\title{
Impact of long- and short-range fiber depletion on the cognitive deficits of fronto- temporal dementia
}

Melissa Savard MSc ${ }^{1,2}$, Tharick A. Pascoal MD, $\mathrm{PhD}^{1}$, Thijs Dhollander, $\mathrm{PhD}^{3}$, Yasser IturriaMedina PhD ${ }^{4}$, Paolo Vitali, MD, PhD, FRCPC ${ }^{5}$, Joseph Therriault BA ${ }^{1}$, Sulantha Mathotaarachchi $\mathrm{MSc}^{1}$, Andrea L. Benedet ${ }^{1}$, Serge Gauthier MD ${ }^{6}$, and Pedro Rosa-Neto MD, PhD ${ }^{1,4,6}$ for the Frontotemporal Lobar Degeneration Neuroimaging Initiative*

${ }^{1}$ Translational Neuroimaging Laboratory, The McGill University Research Centre for Studies in Aging, Montreal, Canada

${ }^{2}$ Douglas Hospital Research Centre, McGill University, Montreal, Canada

${ }^{3}$ Developmental Imaging, Murdoch Children's Research Institute, Melbourne, Australia

${ }^{4}$ Montreal Neurological Institute, McGill University, Montreal, Canada

${ }^{5}$ Department of Neurology and Neurosurgery, McGill University, Montreal, Canada

${ }^{6}$ Department of Psychiatry, McGill University, Montreal, Canada

* Data used in preparation of this article were obtained from the Frontotemporal Lobar Degeneration

Neuroimaging Initiative (FTLDNI) database (http://4rtni-ftldni.ini.usc.edu/). Data collection and sharing for this project was funded by the National Institutes of Health (Grant R01 AG032306). The investigators at NIFD/FTLDNI contributed to the design and implementation of FTLDNI and/or provided data but did not participate in analysis or writing of this report.

\begin{abstract}
Fronto-temporal dementia (FTD) is a neurodegenerative disease characterized by focal atrophy of the gray matter (GM), especially in the frontal and temporal regions. Recent studies suggest a framework where white matter (WM) atrophy plays an important role in FTD pathophysiology. However, these studies often overlook the fact that WM tracts bridging different brain regions may have different vulnerabilities to the disease and the relative contribution of GM atrophy to this WM model, resulting in a less comprehensive understanding of the relationship between clinical symptoms and pathology.
\end{abstract}


Here, by leveraging the sensitivity of advanced diffusion MRI modelling and metrics to precise white matter microstructural properties, we aim to clarify the relative contributions of WM fibers and GM atrophy to the cognitive symptoms typically found in FTD. A total of 155 participant from the Frontotemporal Lobar Degeneration Neuroimaging Initiative (FTLDNI) were analysed, including 68 normal elderly controls $(\mathrm{CN}), 28$ behavioral variants $(\mathrm{BV}), 26$ sematic variants $(\mathrm{SV})$ and 30 progressive non fluent aphasia variants (PNFA) of FTD. Diffusion MRI analysis was performed using two complementary techniques: whole brain fixel-based analysis (FBA) and structural connectivity based on probabilistic tractography. Whole brain GM atrophy was assessed using voxel-based morphometry (VBM). Using a common factor analysis to extract a semantic and an executive factor, we aim to test the relative contribution of WM and GM of specific tracts in predicting cognition. We found that semantic symptoms were mainly dependent on short-range WM fiber disruption, while damage to longrange WM fibers was preferentially associated to executive dysfunction with the GM contribution to cognition being predominant for local processing. Our results support the importance of the disruption of specific WM tracts to the core cognitive symptoms associated with FTD. As large-scale WM tracts, which are particularly vulnerable to vascular disease, were highly associated with executive dysfunction, our findings highlight the importance of controlling for risk factors associated with deep white matter disease, such as vascular risk factors, in patients with FTD in order not to potentiate underlying executive dysfunction. 


\section{Introduction}

Frontotemporal dementia (FTD) is the second most prevalent form of early onset dementia (Bang, Spina, \& Miller, 2015; Cairns et al., 2007). The misfolding and aggregation of proteins such as tau, TDP-43, FUS or ubiquitin-positive proteins encompass nearly all cases of FTD (Seelaar, Rohrer, Pijnenburg, Fox, \& Van Swieten, 2011). FTD clinical phenotype includes behavioural, executive and language dysfunction without primary amnesia. The initial clinical manifestation of the disease characterizes FTD cases into 3 common variants: the behavioural (BV) and two distinct forms of primary progressive aphasias, the semantic (SV) and the progressive non-fluent aphasia (PNFA). As the disease progresses both language and behavioural dysfunction may appear in all variants.

While FTD has long been considered a grey matter (GM) disease, recent advance in diffusion MRI (dMRI) research has revealed that white matter (WM) is also much involved in the pathophysiology of the disease. GM atrophy is typically observed in the insula (all variants), the bilateral anterior cingulate and frontal lobe $(\mathrm{BV})$, left anterior temporal lobe $(\mathrm{SV})$ and left premotor and inferior frontal cortex (PNFA). Widespread WM abnormalities have been observed in the uncinate fasciculus, superior frontal, inferior frontal and inferior fronto-occipital fasciculi, the corpus callosum and the cingulum bundle, with a large overlap amongst participants; see (Greaves \& Rohrer, 2019; Meeter, Kaat, Rohrer, \& Van Swieten, 2017; Rohrer et al., 2010) for recent reviews of MRI findings in FTD. In some mutation carriers, WM changes are detectable up to 30 years before symptoms onset (Jiskoot et al., 2018), strengthening the importance of considering WM alteration as part of the pathophysiology of FTD.

Although often studied separately, WM and GM impairments are not occurring in isolation from one another. Alteration from GM may propagate to WM and, reciprocally, WM damage may propagate to GM via Wallerian degeneration or retrograde degeneration (Metzler-Baddeley et al., 2019; N. Villain et al., 2008; Nicolas Villain et al., 2010). The aforementioned constructs suggest a dynamical and interdependent relationship between GM and WM as determinants of cognitive symptoms in neurodegenerative conditions such as FTD. Despite strong evidence of isolated effects of both WM and GM disruptions on FTD, their relative contribution to the impairment of the different cognitive domains typically affected in patients with FTD is unknown. 
In the present study, we aim to clarify the relative contribution of different properties of WM fibers and GM to the cognitive impairment (semantic and executive) in FTD patients. Specifically, we used a WM fixel-based analysis (FBA) combined with a structural connectivity and GM voxel-based morphometry analyses to 1) provide an improved characterisation of the whole brain fixel density (FD) and crosssection (FC) impairment across the variants 2) investigate the relationship between the WM metrics and GM volume, 3) evaluate the relationship between WM metrics and cognition domains in patients and 4) test the relative contribution of WM and GM of specific tracts in predicting cognition. We studied these associations across different WM tracts under the assumption that these associations vary depending on specific WM tracts characteristics. We found that semantic symptoms were mainly dependent on short-range WM fiber disruption, while damage to long-range WM fibers was preferentially associated to executive dysfunction with the GM contribution to cognition being predominant for local processing.

\section{Methods}

\section{Study Sample}

All data were obtained from the Frontotemporal Lobar Degeneration Neuroimaging Initiative (FTLDNI), through the LONI portal (http://adni.loni.usc.edu). FTLDNI is a multicentric longitudinal database, collecting MRIs, PET and CSF biomarkers in FTD patients and age-matched controls. All patients were clinically diagnosed by a multidisciplinary consensus panel (Ljubenkov et al., 2018; Staffaroni et al., 2019). For the present analysis, we included a total of 155 participants with crosssectional DWI sequence passing quality control. The dataset comprised 68 Normal Elderly Control (CN), 28 Behavioural Variants (BV), 30 Progressive Non-Fluent Aphasia (PNFA) and 26 Semantic Variant (SV) FTD patients.

\begin{tabular}{lllll}
\hline & CN $(\mathbf{N}=\mathbf{6 8})$ & BV $(\mathbf{N}=\mathbf{2 8})$ & SV $(\mathbf{N}=\mathbf{2 6})$ & PNFA $(\mathbf{N}=\mathbf{3 0})$ \\
\hline Age (year) & $61.8(8.2)$ & $60.6(6.4)$ & $62.6(6.0)$ & $68.3(7.4)$ \\
Sex (female) & $60.3 \%$ & $21.4 \%$ & $42.3 \%$ & $63.3 \%$ \\
CDR language & - & $0.84(0.53)$ & $1.04(0.47)$ & $1.38(0.66)$ \\
CDR behaviour & - & $1.48(0.72)$ & $0.98(0.48)$ & $0.41(0.46)$ \\
CDR sum boxes & - & $5.96(2.78)$ & $3.54(2.02)$ & $1.59(1.55)$
\end{tabular}

Table 1. Demographics 


\section{MRI acquisition}

A total of 65 volumes (diffusion-weighted images for 60 gradient directions at $\mathrm{b}=2000 \mathrm{~s} / \mathrm{mm}^{2}$ and 5 images at $b=0 \mathrm{~s} / \mathrm{mm}^{2}$ ) were acquired on a Siemens Trio Tim with the following parameters: repetition time $/$ echo time $=6600 / 86 \mathrm{~ms}, 2.2 \mathrm{~mm}$ isotropic voxels, phase encoding direction $=$ AP. A 3D MPRAGE image $(1 \mathrm{~mm}$ isotropic voxels, repetition time $/$ echo time $=2300 / 2.98 \mathrm{~ms}$ and flip angle $=9$ degrees) was also used to measure GM volume.

\section{dMRI processing}

All dMRI data were preprocessed using MRtrix3 (J. D. Tournier et al., 2019). Preprocessing steps included denoising (Veraart et al., 2016), Gibbs ringing correction (Kellner, Dhital, Kiselev, \& Reisert, 2016), eddy-current and motion correction (Andersson \& Sotiropoulos, 2016) and bias field correction (Tustison et al., 2010). Response functions for single-fibre WM as well as GM and CSF were estimated from the data themselves using an unsupervised method (Dhollander, Mito, Raffelt, \& Connelly, 2019). Single-Shell 3-Tissue CSD (SS3T-CSD) was performed to obtain WM-like FODs as well as GM-like and CSF-like compartments in all voxels (Dhollander \& Connelly, 2016), using MRtrix3Tissue (https://3Tissue.github.io), a fork of MRtrix3 (J. D. Tournier et al., 2019). The resulting WM-like FOD, GM-like and CSF-like images were used to perform multi-tissue informed log-domain intensity normalisation (Figure 1a). A cubic b-spline interpolation was used to upsample the WM FOD images to $1.3 \mathrm{~mm}$ isotropic voxels. A study specific template was created using the WM FOD images from $30 \mathrm{NC}$ to which all subjects FOD images were subsequently non-linearly registered (D. Raffelt, Tournier, Crozier, Connelly, \& Salvado, 2012; D. Raffelt et al., 2011). Finally, the WM FOD template was used to generate a whole brain probabilistic tractogram (J.-D. Tournier \& , F. Calamante, 2010) which was then filtered from 20 million tracts to 2 million tracts to reduce reconstruction bias (Smith, Tournier, Calamante, \& Connelly, 2013, 2015b).

\section{Fixel-based metrics}

We used the fixel-based analysis framework (D. A. Raffelt et al., 2017; D. Raffelt, Tournier, Rose, et al., 2012) to compute the fiber density (FD) and the fiber cross-section (FC) at the fixel level (Figure 1b). A "fixel" here refers to a "fiber population in a voxel"; hence, when multiple fibers are crossing in 
the same voxel, they each still have individual measures of FD and FC. Interestingly these metrics provide complementary information about the white matter. Namely, FD-based differences can be interpreted as intra-axonal microstructural alterations, while FC-based differences can be attributed to macroscopic changes of a fiber bundle, i.e a tract that is atrophied or hypertrophied in respect to the WM FOD template.

\section{Structural connectivity analyses}

A probabilistic tractography algorithm (J.-D. Tournier \& , F. Calamante, 2010) with dynamic seeding (Smith, Tournier, Calamante, \& Connelly, 2015a) was used to generate 20 million tracks for each participant WM FODs in the template space (Figure 1c). The tractogram was subsequently filtered using SIFT (Smith et al., 2013) until the algorithm reaches convergence. We used the Desikan-Killiany GM atlas (DKT) to compute the amount of fibers connecting 78 grey matter regions (Desikan et al., 2006). An affine transformation was first calculated from the MNI ICBM152 WM parcellation to the diffusion template space (Figure 1e). The affine transform was applied to the DKT atlas to bring it in diffusion template space (Figure 1f) and the atlas was corrected by the amplitude of the template WM FOD, where amplitudes higher than 0.1 were set to zero. A visual inspection of the resulting GM atlas insured that all GM regions were well represented. Structural connectomes were calculated as the total number of fibers paths connecting each pair of GM regions (Figure 1g). The results of the statistical analysis performed on the connectomes were visualized using BrainNet Viewer (Xia, Wang, \& He, 2013).

\section{GM voxel-based morphometry}

T1 anatomical images were segmented in GM, WM and CSF tissue probability images using the SPM12 segmentation tool (https://www.fil.ion.ucl.ac.uk/spm/doc/biblio/). A study specific brain template was then calculated using the GM and WM probabilities from 30 normal elderly control using the Dartel toolbox (Ashburner, 2007). Each individual GM map was non-linearly registered to the CN template (Figure 1d). GM probabilities were modulated and filtered using a full width half maximum of $8 \mathrm{~mm}$. Intracranial volume (ICV) was defined as the sum of GM, WH and CSF probabilities images in native T1 space. 


\section{Cognitive tests}

In order to clinically characterize the FTD patients, the following cognitive scores were used: the total correct immediate $(30 \mathrm{sec}$.$) and delayed (10 \mathrm{~min})$ items recall of the California Verbal Learning Test, the total Boston naming correct score, the sematic verbal fluency (animal), the phonemic verbal fluency (d words), the modified trail making completion time, the forward and backward digit span. A maximum likelihood common factor analysis ("factoran" function in Matlab, with varimax rotation) was used to obtain a parsimonious representation of all available cognitive scores, as we wanted to obtain an explanatory model for the correlations among these scores. A two common factor hypothesis was first rejected (approximate chi-squared test; $\mathrm{p}<0.05$ ) while a 3 factor model fails to reject the null hypothesis (approximate chi-squared test; $\mathrm{p}<0.42$ ), suggesting that the latter model provides a satisfactory explanation of the covariation in these data (see Figure 5a for the factor loadings results). The factor scores were calculated using a weighted least score estimate.

\section{Tract of interest analysis}

Selected connectivity-based pairs of GM regions were used to extract the tracts connecting them, which allows to investigate the tract specific relations between GM volume, FD and FC and cognition. Using the template filtered tractogram (2M streamlines), we extracted the streamlines assigned to the pair of selected GM regions (Figure 1i). The resulting streamlines were then automatically thresholded into a binary fixel mask using an automated optimal threshold (Ridgway et al., 2009). The connectivity-based FC and FD values were then averaged in the mask. The GM volume was assessed by calculating the average GM VBM values of the connecting regions (see Figure 6a-b for a graphical representation). To investigate all the selected connections as a whole, we standardized GM, FC and FD across all connections and used repeated general linear models (GLM) to obtain the prediction estimate for their respective cognitive factors adjusted for age, sex and ICV (Figure 1i). Finally, we calculated the average streamline length for each tract, which best represents the overall length of the tract. 


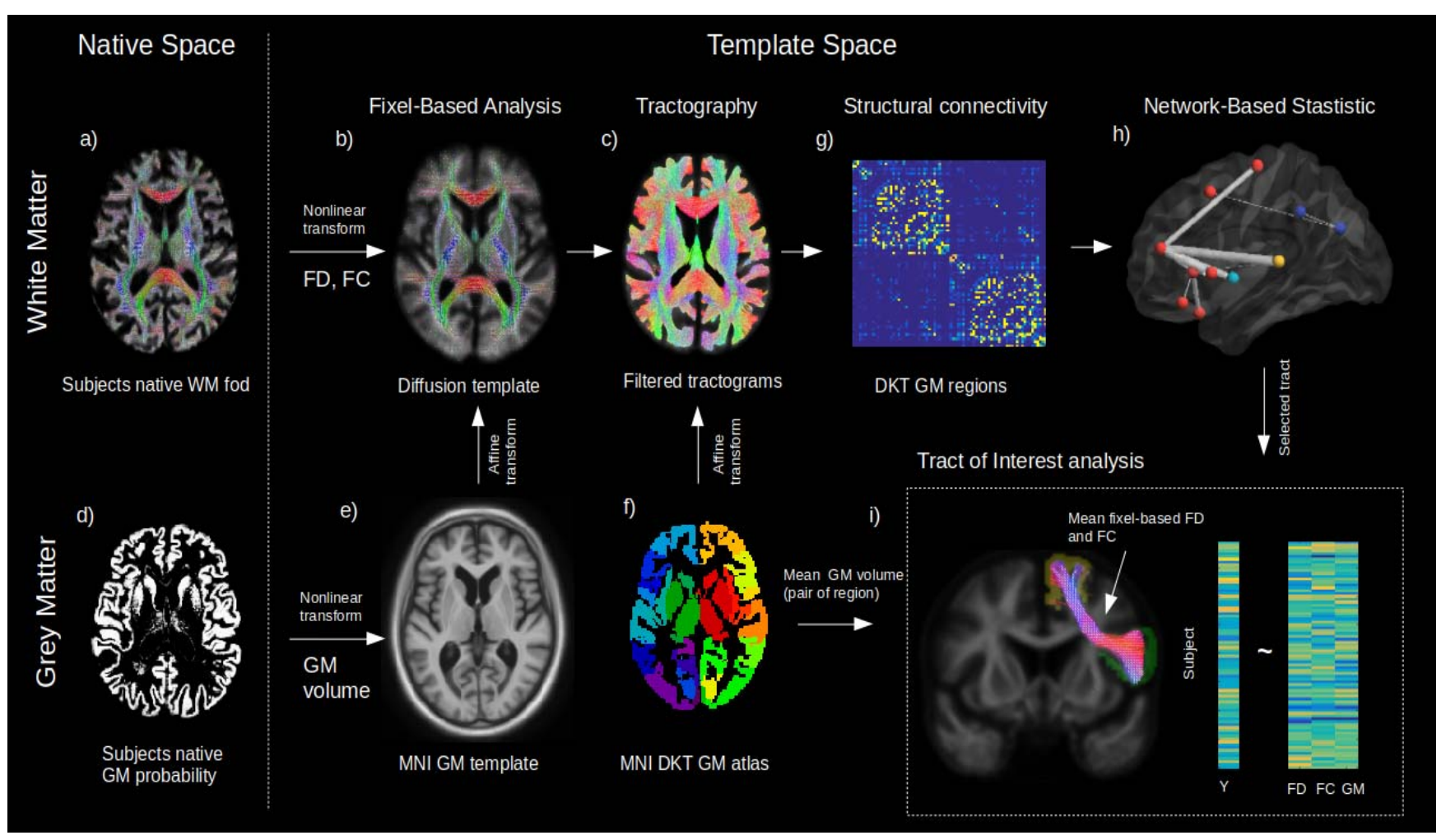

\section{Figure 1. Method workflow}

The main steps of the methods are shown form the native space (left) to template space (right). For the white matter (upper panels), native diffusion weighted MRIs were first preprocessed to obtain individual normalized WM FODs (a). WMFODs, were non linearly registered to a study specific WM FOD template (b), to obtain the fiber density (FD) and fiber cross-section metrics (FC), later used in whole rain Fixel-Based analysis. The template space WM FODs were then used to generate individual probabilistic tractograms (c). For the grey matter (lower panels), native space GM probability maps were warped to a study specific GM template in MNI space to obtain individual template space GM volume (e). An affine transform was estimated between MNI template and the diffusion template space which was subsequently applied to the DKT grey matter atlas to bring the DKT atlas in diffusion space (f). Individual structural connectivity matrices were then obtained by counting the amount of fibers connecting each pair of GM regions within the DKT atlas (g). Significant difference in connectivity for a given dependant variable (Y) were then tested using the Network Based Statistic enhanced (h). Significant predictors (connections) were selected to access the relative importance of GM volume and WM (FD and FC) within each connection in predicting Y (h), where mean FD and FC were obtained in fixels belonging to the connection streamlines and GM was the average of both GM regions volume for each subject. 


\section{Statistical Analysis}

Fixel-wise whole brain characterisation of the relationship between FC, FD, diagnosis and cognition was carried out using the connectivity-based fixel enhancement (CFE) method (D. A. Raffelt et al., 2015). For the structural connectivity analysis, a common connectivity mask was generated for the top $20 \%$ connections of the population template. Relationship between the connectivity, diagnosis and cognition was calculated using the network-based statistical enhancement method (Vinokur, Zalesky, Raffelt, Smith, \& Connelly, 2015). For both methods, family wise corrected p-values were obtained via permutation testing $(\mathrm{n}=1000)$. VBM analyses were performed using VoxelStats (Mathotaarachchi et al., 2016). Correction for multiple comparisons was performed using random field theory with a cluster threshold (after correction) of $\mathrm{p}<0.01$. Analyses of cognition were performed in patient groups only. All statistical models were corrected for age, sex and ICV.

\section{Results}

\section{Fiber loss in FTD variants}

Variants of FTD all had extensive WM impairments compared to normal controls after correction for age, sex and ICV. Figure 2 a-c shows the streamline segments associated to significantly reduced FC and FD (FWE-corrected P-value < 0.05; color coded by direction) for the BV, PNFA and SV respectively. Irrespective of the variant, reduced FC (Figure 2 a-c upper panels) could be observed in large associative fibers including the uncinate fasciculus, the inferior fronto-occipital fasciculus and the superior longitudinal fasciculus, cingulum and corpus callosum. Despite a large common network, variant-specific differences could be noted in the bilateral anterior and medial part of the frontal cortex and lateral orbitofrontal WM for BV (Figure 2a), while PNFA presented with reduced FC in the caudal part (precentral gyrus/supplementary motor area) of the left frontal cortex (Figure 2b) and SV showed a left predominant FC reduction in the inferior longitudinal fasciculus (Figure 2c). Reduced FD patterns (Figure 2 a-c lower panels) were similar to those observed for FC although with a lower spatial extent.

Structural connectivity analysis (Figure 2d-f), although based on a different method (tractography), provided complementary information to the FBA analysis, about specific GM regions that may be 
affected by the WM impairment. Significant reductions (FWE-corrected P-value < 0.001) in tracts connecting GM regions are shown for frontal regions (red), the insula (light blue), the temporal lobe (yellow), subcortical region (green) and parietal regions (dark blue), where the line thickness corresponds to the strength of the effect. Compared to normal controls, BV (Figure 2d) had the largest reduction in bilateral insula - inferior frontal cortex (pars opercularis and triangularis) connectivity, followed by bilateral reduction in thalamo-frontal (rostral middle frontal) connectivity. For PNFA (Figure 2e), the largest reduction was also observed in insula - inferior frontal cortex (pars opercularis and triangularis) connectivity but in the left hemisphere only, followed by precentral - middle frontal connectivity impairment. For SV (Figure 2f), the largest reduction occurred in the left hemisphere between the thalamus and the temporal cortex (superior and middle), but also between the lateral orbitofrontal and superior frontal cortex, followed by intra-temporal connectivity reduction. 


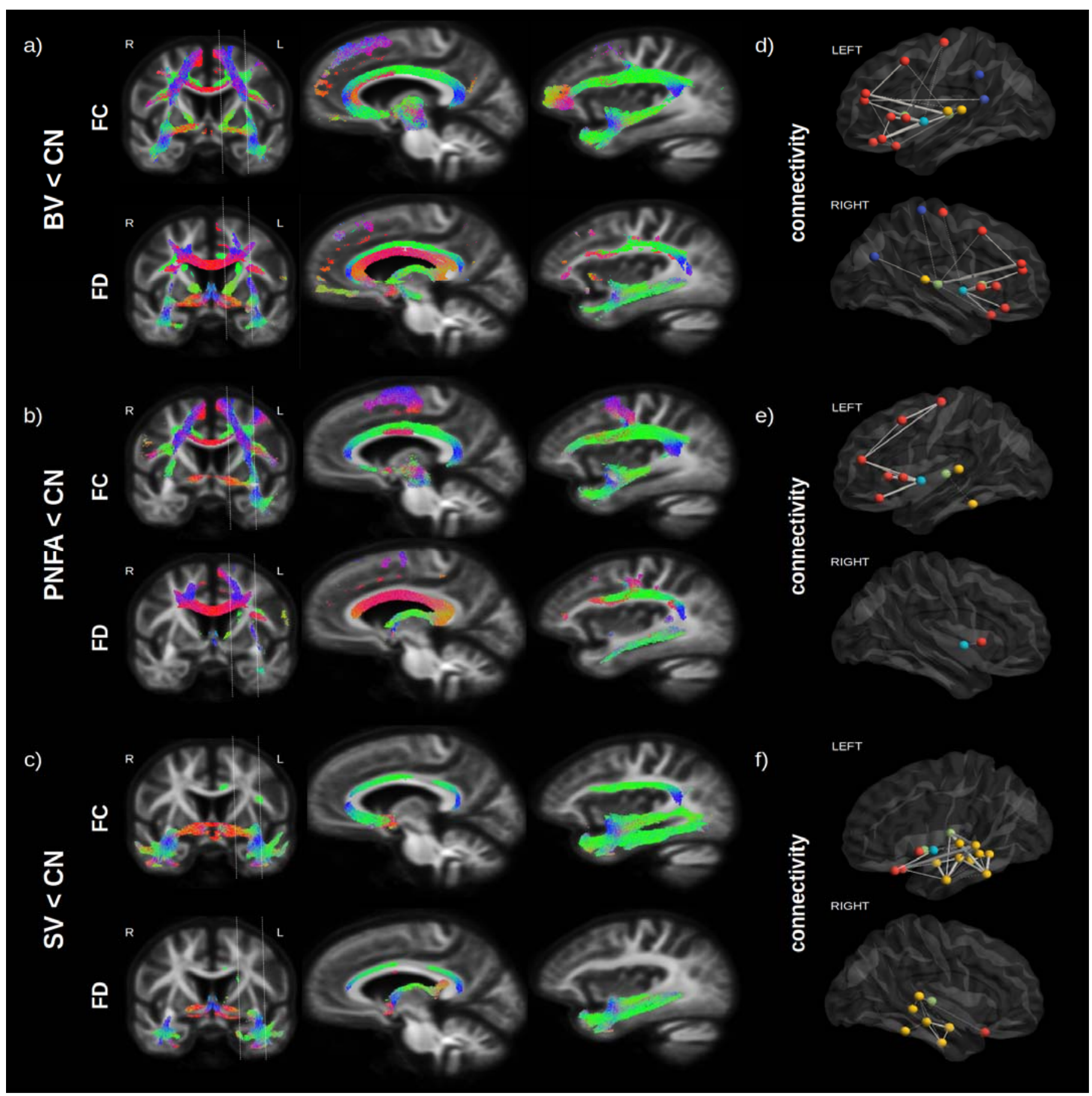

\section{Figure 2. Fiber loss in FTD variants}

Streamlines (color coded by direction) associated to significantly reduced FC and FD (FWE-corrected P-value) are shown for BV vs CN (a), PNFA vs CN (b) and SV vs CN (c). Associated structural connectivity reduction (FWE-corrected P-value $<0.001$ ) is shown in panels (d-f) for the ipsilateral left (upper panel) and right (lower panel) hemisphere, where frontal regions are shown in red, the insula in 
bioRxiv preprint doi: https://doi.org/10.1101/2020.05.01.064576; this version posted May 2, 2020. The copyright holder for this preprint (which was not certified by peer review) is the author/funder, who has granted bioRxiv a license to display the preprint in perpetuity. It is made available under aCC-BY-NC-ND 4.0 International license.

light blue, the temporal lobe in yellow, subcortical regions in green and parietal regions in dark blue.

The line thickness corresponds to the statistical strength of the effect.

\section{GM atrophy in FTD variants}

Significant differences, after correction for age, sex, ICV and multiple comparisons, were observed for GM volume between CN and FTD variants (Figure 3 a-c). BV (Figure 3a) had a widespread reduction in bilateral GM volume with the strongest effect seen in the insula, orbitofrontal, anterior cingulate and prefrontal cortex (middle and inferior) while PNFA (Figure 3b) had a left predominant atrophy in the premotor part of the frontal cortex, the insula and prefrontal cortex (middle and inferior), and SV (Figure 3c) had a bilateral (but left predominant) atrophy of the whole temporal lobes and to a lesser extend insula atrophy. Taken together (Figure 3d), the 3 variants share overlapping GM atrophy in the insula, while BV and SV share atrophy in the temporal lobe and orbitofrontal cortex, and PNFA and BV share atrophy in the left prefrontal cortex (middle and inferior).

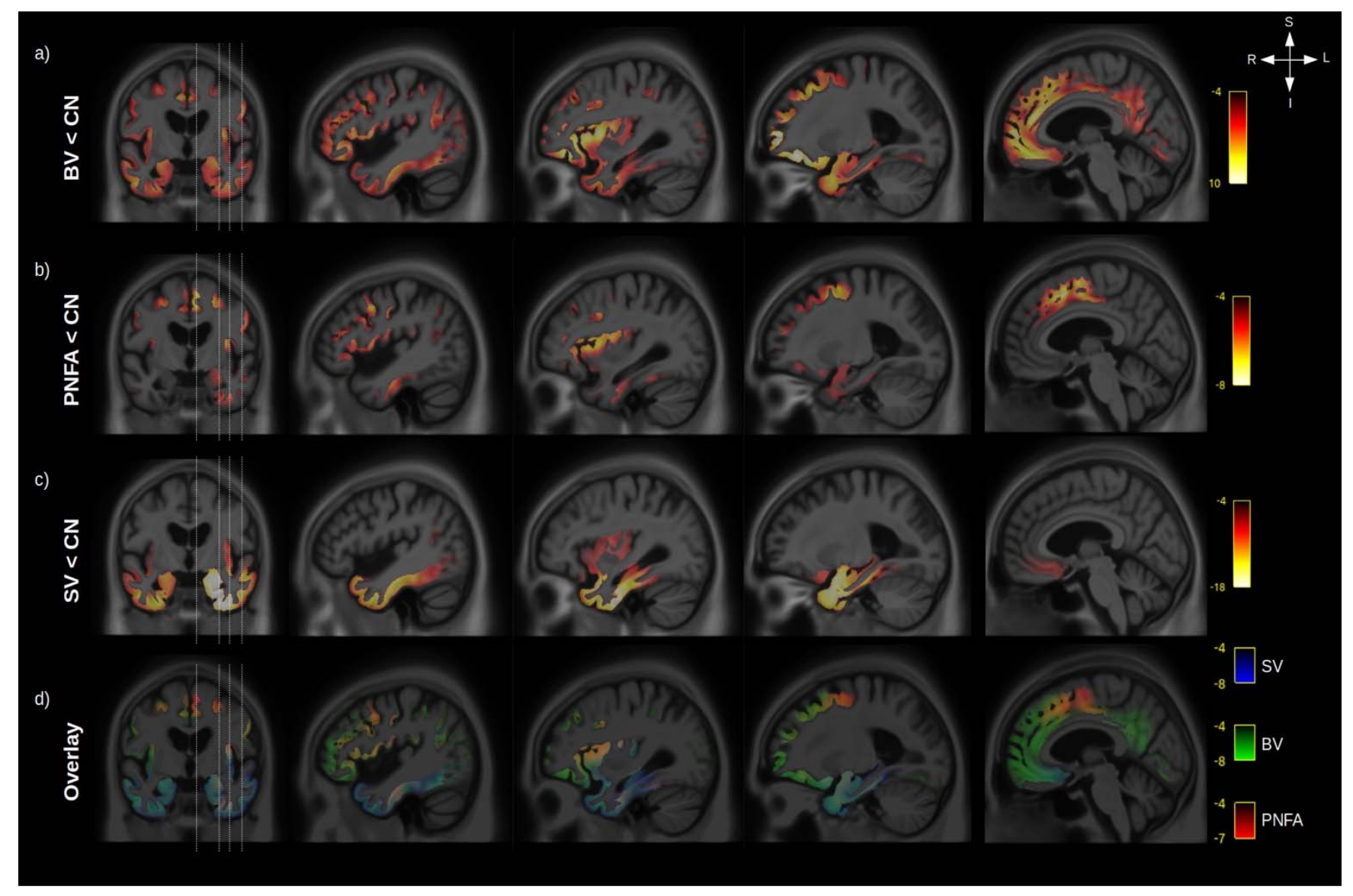

Figure 3. GM atrophy in FTD variants 
Significant (RFT p value <0.05) grey matter volume decrease is shown for BV vs CN (a), PNFA vs CN (b) and SV vs CN (c). Legend are showing the magnitude of the voxelwise T values. An overlay of the statistical maps (d) is shown for BV (green), SV (blue) and PNFA (red), with associated T values color bars.

\section{Relationship between GM atrophy and WM microstructural impairment}

The peak of the maximum GM atrophy for each variant was used as a seed (Figure 4 a-c left panels) to investigate the relationship between GM and whole brain WM fiber cross section and fiber density across all participants. Streamlines associated to significant fixels after correction for multiple comparison are shown for the relationship with FC (middle panels) and FD (right panels). Independently of the seed location, a strong relationship was found between GM atrophy and reduced FC and FD for the inferior fronto-occipital fasciculus, uncinate fasciculus and superior longitudinal fasciculus. GM volume for the BV orbitofrontal seed (Figure 4a) was also associated with the inferior part of the precentral cortex, PNFA premotor seed (Figure 4b) with the precentral WM fibers and SV inferior temporal seed with impairment in the inferior longitudinal fasciculus.

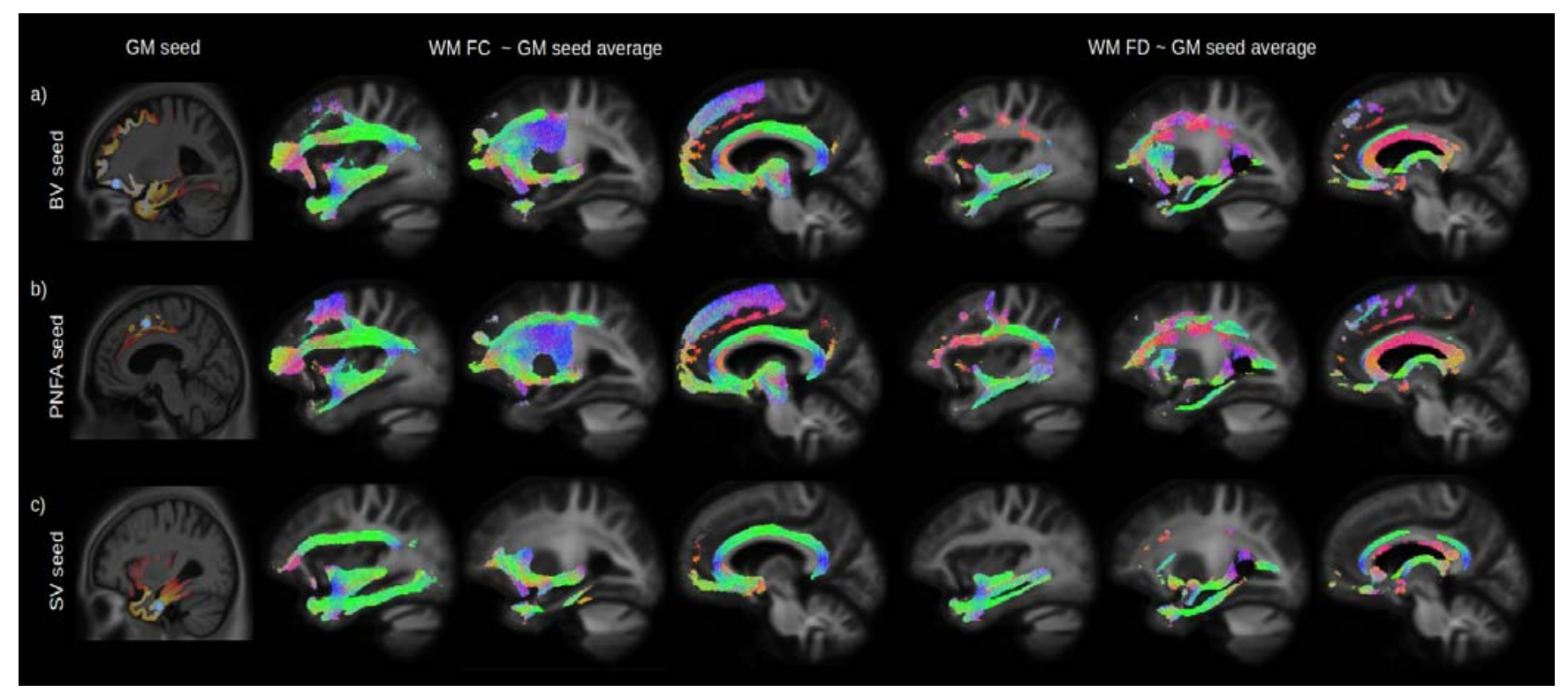

\section{Figure 4. Relationship between GM atrophy and WM microstructural impairment}

The peak of the maximum GM atrophy for each variant (BV, PNFA and SV), was used as a seed (Figure 4 a-c left panels, blue dot) to investigate the relationship between GM and whole brain WM fiber cross section (FC) and fiber density (FD) across all participants. Streamlines associated with 
significant relationships (FWE-corrected P-value < 0.05) between the average GM volume of each seeds are shown for FC (middle panels) and FD (right panels). Streamlines are color coded by direction.

\section{Cognitive domains and WM microstructure}

In order to assess the relationship between impaired cognition and WM fiber density (FD) and fiber cross-section (FC), we first performed a common factor analysis across eight cognitive scores in patients only. Cognitive scores could be represented by 3 principal factors (Figure 5a). The first factor (F1) was mainly related to semantic processing and comprised naming, category fluency and verbal learning scores (delayed and immediate recall). The second factor (F2) was mainly related to executive processing and comprised digit span, trail making and letter fluency scores. The third factor (F3) was only related to verbal fluency (category and letter). Although SV patients had on average a lower semantic factor score compared to BV $(\mathrm{p}=0.034)$ and PNFA $(\mathrm{p}<0.001)$ and a higher executive factor score ( $<$ 0.001; vs BV and PNFA), a large within group variability can be noted for all factors (Figure $5 b)$. Patients factor scores were used to investigate the relationship between each cognitive domain and structural connectivity as well as WM metrics FD and FC. After correction for age, sex, ICV and multiple comparisons, the first (semantic) factor was significantly associated to FC in the uncinate fasciculus, the inferior fronto-occipital fasciculus, and the inferior longitudinal fasciculus (Figure 5d; upper pannel). The semantic factor was also associated to reduced connectivity between the left GM temporal regions amongst themselves but also with the supramarginal, lateral orbitofrontal gyrus and with the thalamus (Figure 5c). The second (executive) factor was significantly associated with a reduced FC in the superior longitudinal fasciculus, superior corona radiata, body of the corpus callosum, inferior frontal and precentral WM and in fibers corresponding to the aslant tract (Figure 5d; lower panel). Reduced structural connectivity was predominantly observed between the left superior frontal gyrus and other GM frontal regions (pars orbitalis, pars triangularis, lateral orbitofrontal, rostral middle frontal and precentral gyrus), accompanied by a reduced connectivity between left superior frontal gyrus and other cortices (insula, the superior temporal gyrus and between the inferior parietal cortex) (Figure 5c). Although not shown in the figure, FD yielded similar spatial relationships than FC. No significant relationship could be found between the third factor (verbal fluency) and FC, FD or structural connectivity (not shown). 


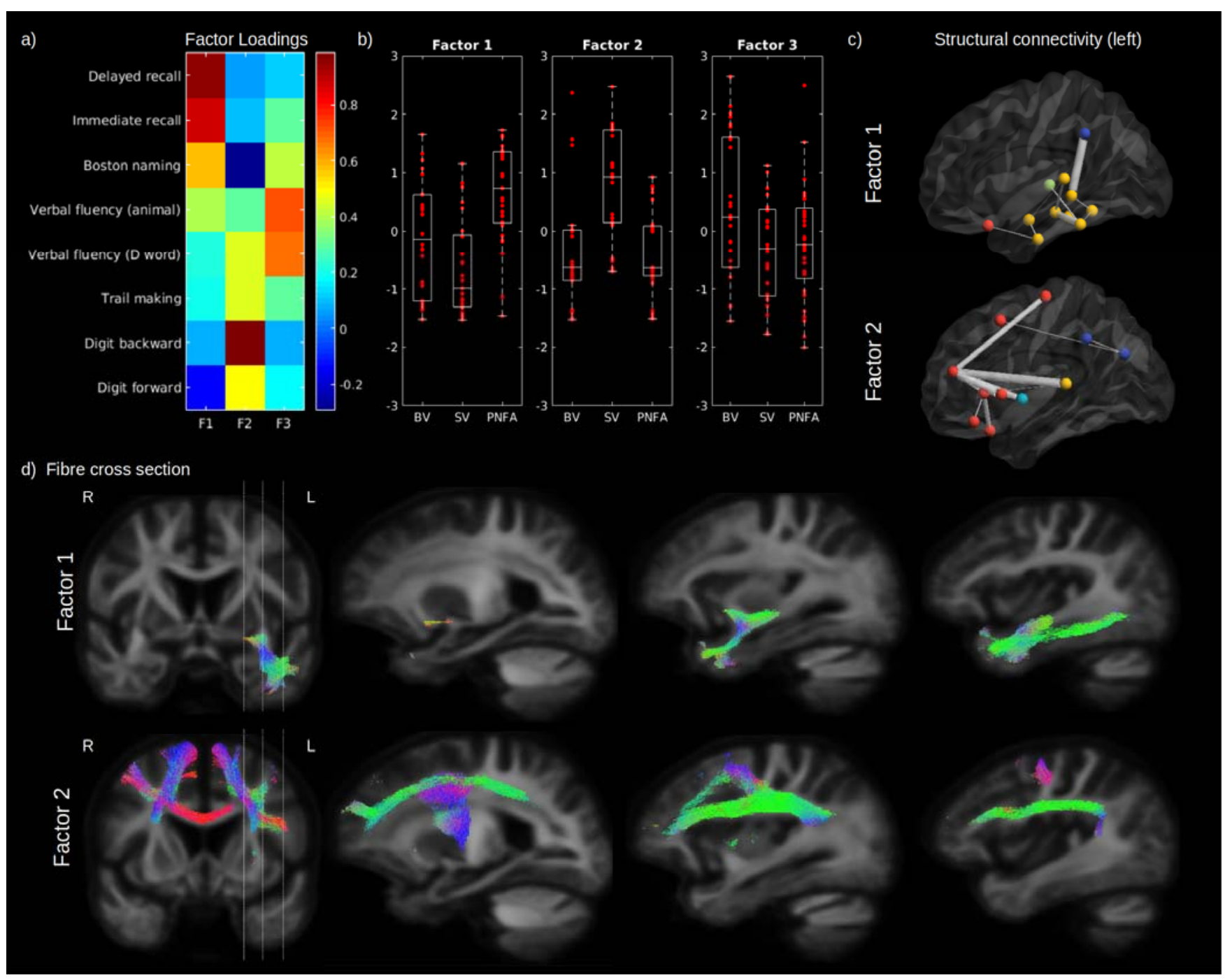

\section{Figure 5. Cognitive domains and WM microstructure}

The factor loadings for the common factor analysis of selected cognitive tests are shown in (a). Associated factors scores are shown in (b) for BV, SV and PNFA, for Factor 1 (left panel), Factor 2 (middle panel) and Factor 3 (right panel). Significantly reduced structural connectivity (across all patients ; FWE-corrected P-value < 0.01) is shown in (c) for the Factor 1 (upper panel) and Factor 2 (lower panel), for the ipsilateral left (upper panel) hemisphere connectivity, where frontal regions are shown in red, the insula in light blue, the temporal lobe in yellow, subcortical regions in green and parietal regions in dark blue. The line thickness corresponds to the statistical strength of the effect. Significant relationship (FWE-corrected P-value < 0.05) between the cognitive factors and FC is shown in (d) with associated streamlines (color coded by direction) for Factor 1 (upper panel) and Factor 2 (lower panel). 


\section{Relative contribution of GM and WM to predict cognitive impairment}

In order to disentangle the contribution of WM and GM abnormalities on cognition impairment, we used the connectivity-based pair of GM regions previously associated to identified cognitive factors (F1 and F2 ; FEW-p < 0.001), where VBM-derived GM volume was averaged for the pair of regions and FD and FC averages were calculated from the fixel associated to the connecting streamlines (see tract of interest method section). Example of pair of GM regions and fixel mask are shown in Figure 6 a-b for the factors 1 (semantic) and 2 (executive) respectively. After correcting for age, sex and ICV, GM volume and FC better explained the variance for the first cognitive factor $(39.2 \%$ and $32.2 \%$, respectively) than FD (12.7\%) (Figure 6a right panels). On the other hand, for the second factor, FC explains the variance better $(31.7 \%)$ than GM (18,5\%) and FD (18.9\%) (Figure 6b right panels). The comparisons of all the selected connection standardized estimates revealed that, for most connections, GM, FD and FC could predict the cognition (Figure 6c; left panel). Importantly, when included together in the same GLM (Figure 6c; right panel), the contribution of the WM was reduced but not for all connections. We then tested the relationship between the standardized estimates and the average fiber length and found a positive relationship for FD (Figure 6d ; left panel, p=0.006) and a trend level negative relationship for GM (Figure 6d ; right panel, $\mathrm{p}=0.058$ ), but not for FC (Figure 6d middle panel, $\mathrm{p}=0.94)$.
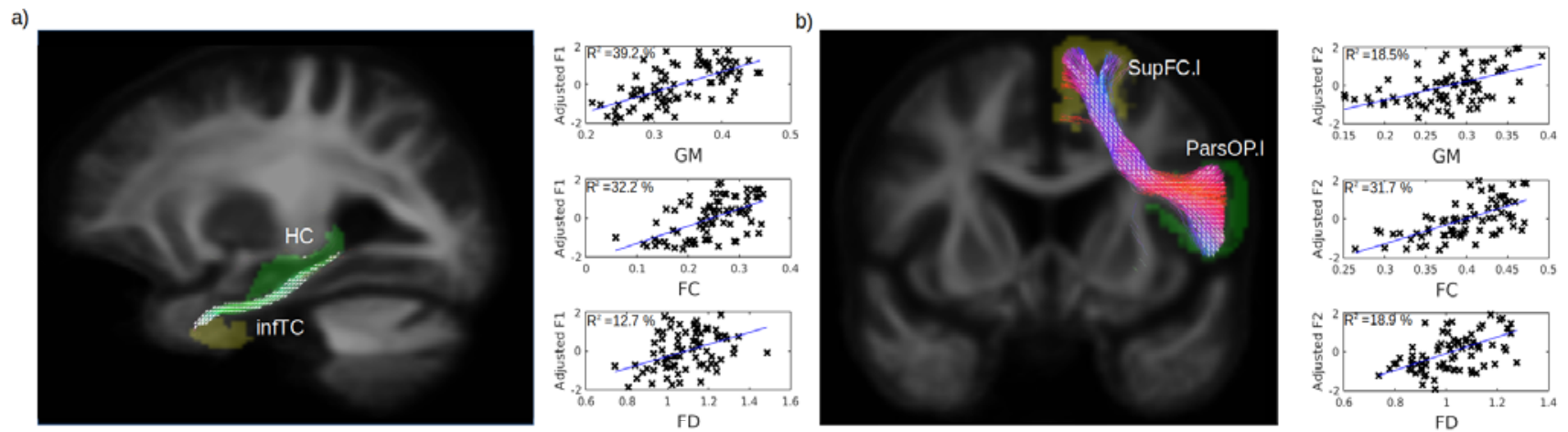

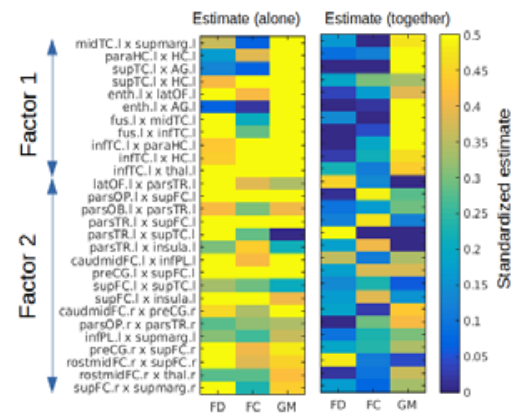

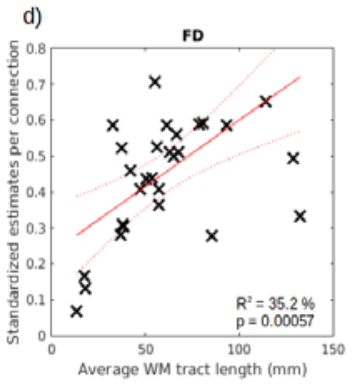
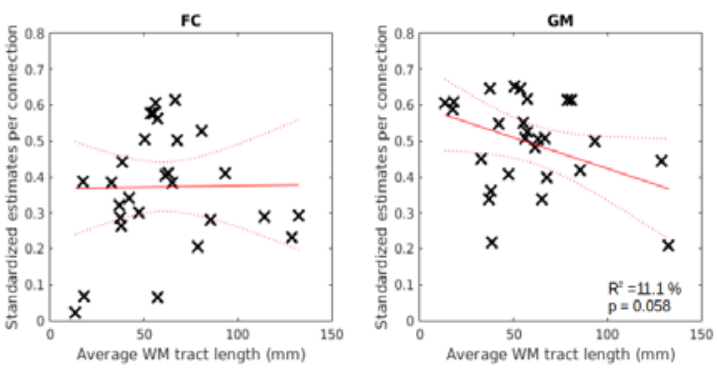


\section{Figure 6. Respective contribution of GM and WM to predict cognitive impairment}

Example tract of interest, selected from the significant relationship between structural connectivity and cognitive Factor, are shown in (a-b), for Factor 1 and 2 respectively. The pair of connecting cortical regions (green and yellow) and the fixel binary mask extracted from the streamlines connecting the pair of GM regions (white) are used to calculate the connection specific relationship across subjects between the cognitive factors and the average regional GM volume (upper panel) and the average fiber cross-section (FC) and fiber density (FD) in the fixel binary mask (middle and lower panel respectively). The standardized estimate of the relationship between the cognitive factors 1 and 2 is shown in (c) for all selected connections, where the left panel shows the values when FD, FC and GM were use as single predictors and the right panels show the partial estimates when FD, FC and GM were all included together in the model. The relationship between each connection estimate and their associated average fiber length is shown in (d) for FD (left panel), FC (middle panel) and GM (right panel).

\section{Discussion}

In this study we aimed to quantify the relative contribution of WM and GM abnormalities as determinants of cognitive impairment in FTD clinical spectrum. We found that, although regional differences in WM properties were observed in all variants, all FTD cases had fiber density or crosssection abnormalities across a large WM network connecting the frontal and temporal cortices. Furthermore, these WM abnormalities were linked to patterns of GM atrophy and cognitive decline across FTD variants. The differential contributions of WM and GM on cognition depended on the length of WM fibre. Although both GM and WM abnormalities contribute to FTD symptoms, these results highlight the importance of WM fiber density and cross-section in FTD pathophysiology.

\section{FD and FC and structural connectivity phenotypes in variants of FTD}

Our study identified a large network (uncinate fasciculus, superior longitudinal fasciculus, inferior fronto-occipital fasciculus, cingulum and corpus callosum) of white matter impairment being shared across FTD variants, extending findings from previous reports (Federica Agosta et al., 2015). Also in line with previous literature, we found SV to have additional impairments in the inferior frontooccipital fasciculus (Acosta-Cabronero et al., 2011; Federica Agosta et al., 2010; Galantucci et al., 2011; Matsuo et al., 2008; Whitwell et al., 2010) , BV in the frontal cortex (F. Agosta et al., 2012; 
Mahoney et al., 2014; Piguet, Hornberger, Mioshi, \& Hodges, 2011; Yu \& Lee, 2019; Zhang et al., 2009) and PNFA in SMA white matter fibers (Federica Agosta et al., 2015; Mahoney et al., 2013). Our structural connectivity results were in agreement with the fixel based results suggesting that both techniques were able to detect WM impairments in FTD. In addition, we found that one of the largest reductions in structural connectivity was between thalamo-frontal regions, supporting the finding that thalamic atrophy is a prominent feature of FTD (Diehl-Schmid et al., 2019) and that it is common across episodic and genetic mutation (Bocchetta et al., 2018).

\section{Cognitive factor across variants}

Across the FTD spectrum, we found that a common semantic factor explained the variance of scores in the immediate and delayed verbal memory test, picture naming and categorical verbal fluency. This is in line with several studies showing poorer lexical retrieval of semantically degraded words versus semantically intact words (Jefferies, Jones, Bateman, \& Lambon Ralph, 2004; Knott, Patterson, \& Hodges, 2000; Patterson, Graham, \& Hodges, 1994), suggesting that semantic information contributes significantly in (phonological) lexical activation. The second cognitive factor (executive) explained the variance of scores in the modified trail making processing time, digit span (forward and backward) and phonemic, but not categorical, verbal fluency. TMT measures multiple executive functions, including attention, processing speed, set-shifting and digit span (forward and backward) is typically used as an attentional/working memory measure, while phonemic verbal fluency contains both a working memory/executive and a language component. A third factor grouped the two verbal fluency tests together, however this factor only partly explains the variance of each test as they also weighed on semantic processing (for category fluency) and executive functioning (letter fluency), supporting the dual nature of the verbal fluency test (Whiteside et al., 2016) even in non-demented individuals.

\section{WM and cognition in FTD}

\section{Semantic processing}

In the present study, we found evidence of the relationship between semantic deficits and WM impairment in the left uncinate fasciculus, inferior longitudinal fasciculus and inferior fronto-occipital fasciculus, across all variants. The uncinate fasciculus (connecting the orbitofrontal cortex to the temporal pole) have been associated with semantic processing in many studies ; see (Papagno, 2011) for a review. Brain stimulation studies (Duffau, Gatignol, Mandonnet, Capelle, \& Taillandier, 2008; 
Duffau et al., 2005) and post-mortem fiber dissection studies (Martino, Brogna, Robles, Vergani, \& Duffau, 2010) have linked the ventral subcomponent of the inferior fronto-occipital fasciculus, (connecting the frontal lobe to occipital associative extrastriate cortex and the temporo-basal region) and semantic processing. Prior studies led to inconsistent results regarding the involvement of the inferior longitudinal fasciculus, connecting the ventro-anterior temporal lobes, to several occipital regions (fusiform gyrus, lingual gyrus and dorsolateral occipital cortex); see (Cocquyt et al., 2020) for a recent review. Our structural connectivity analysis revealed that the connectivity between the left inferior temporal cortex and the thalamus may also be involved in semantic processing. In general, our findings support the so-called hub model for the semantic processing where the anterior temporal pole represents a unique trans-modal hub receiving and assembling information from different modality specificity brain regions via specific WM connections (Patterson, Nestor, \& Rogers, 2007). Interestingly, it was also proposed (Ralph, Jefferies, Patterson, \& Rogers, 2016) that graded deficit in semantic processing is dependent on the white matter fasciculi connecting the anterior temporal lobe to the cortex, where the uncinate fasciculus, superior longitudinal fasciculus and inferior fronto-occipital fasciculus would convey either social, verbal or visual semantic processing respectively. Altogether, our findings support the hub hypothesis and suggest that it could be expanded further by considering subcortical contribution to the model.

\section{Executive processing}

Executive processing is a prominent frontal function and not surprisingly it is severely affected in the FTD clinical spectrum. We found that the executive function impairment was associated with disruption of WM tracts in the frontal lobe, specifically in superior longitudinal fasciculus, superior corona radiata, the body of the corpus callosum, inferior frontal and supplementary motor WM and the aslant tract. The superior longitudinal fasciculus (connecting the frontal lobe to temporal and parietal cortices) has previously been associated to processing speed (Turken et al., 2008) and working memory (Rizio \& Diaz, 2016) and impairment of the corana radiata (connecting the prefrontal cortex to the basal ganglia and thalamus) has also been associated to executive dysfunction (Hua et al., 2014; Moeller, Willmes, \& Klein, 2015). Interestingly, the aslant tract (connecting the supplementary motor area with the inferior frontal cortex) has been associated with the self-initiated movement and speech production (Kinoshita et al., 2015) and its integrity correlated with the amount of distortion errors that PNFA patients made in spontaneous speech (Mandelli et al., 2014). Moreover, our results suggest that 
the contribution of WM to executive deficits increases with the length of these WM tracts. Reduced WM integrity in large-scale WM tracts was the major player of executive dysfunction in the FTD population. Interestingly, large-scale WM tracts are also particularly vulnerable to WM vascular disease, as observed in post-mortem studies (O'Brien et al., 2002). Moreover, chronic ischemic microvascular lesions, depicted as diffuse WM hyperintensities in brain MRI scans are independently associated with impairment of executive function (Young, Halliday, \& Kril, 2008). Our results could suggest thus that patients with FTD and compromised large-scale WM fibers might be particularly vulnerable to additional vascular pathology. Thus, our findings highlight the importance for controlling vascular risk factors in FTD patients in order not to potentiate the underlining executive dysfunction.

\section{Relationship between GM, WM and cognition}

As WM and GM impairment are too often considered in isolation, one of the goals of our study was to investigate their relative contribution to neurodegeneration. We found that the magnitude of GM atrophy was strongly related to the impairment of WM networks. This was also observed in both AD and FTD using canonical correlation analysis (Avants, Cook, Ungar, Gee, \& Grossman, 2010). Modeling the combined contribution of GM and WM to cognition is not straightforward because of the lack of spatial overlap between these modalities. To overcome this challenge, we took advantage of a common connectivity space that encompasses both structural connectivity and fiber specific WM pathways. This construct allowed us to select anatomically relevant connections, to extract their average regional GM volume and streamlines-based respective FC and FD for predicting their respective contribution on the cognitive domain. Interestingly, within short connections, the contribution of GM atrophy was dominant, while WM FD gained in importance as a function of fiber length. This finding supports a framework in which cognitive functions involving short-range circuits are mostly affected by local GM atrophy, while cognitive processes mediated by long-range fibers are more vulnerable to WM impairment. Thus, our results support the critical importance of considering both GM and WM alterations for a better understanding of distinctively spatially distributed cognitive alterations in neurodegeneration.

\section{Fixel-Based Analysis applied to FTD}

To assess WM fiber density and cross-section, we used a novel fixel-based approach where individual fiber populations, even within the same voxel, can be assessed independently. Older diffusion tensor 
imaging (DTI) techniques, although historically invaluable in offering the earliest opportunities to noninvasively investigate some microstructural properties of WM and their alteration in aging and disease, suffered from the inability to resolve crossing fibers. It was shown that traditional DTI may lead to artefactual findings in neurodegenerative disorders (Mito et al., 2018; J. D. Tournier et al., 2008), both false positive as well as false negative. This severely limits the extent to which such DTI findings can be interpreted or even safely relied upon. Novel techniques, such as constrained spherical deconvolution (J. D. Tournier, Calamante, \& Connelly, 2007; J. D. Tournier, Calamante, Gadian, \& Connelly, 2004) and Fixel-Based Analysis (D. A. Raffelt et al., 2015) have greatly improved the accuracy of diffusion MRI processing and whole brain statistical analysis. The associated metrics, fiber density (FD) and fiber cross section (FC), were recently proposed to capture different properties of the white matter fiber (D. A. Raffelt et al., 2017). Fiber density is considered a measure of WM microstructure, while FC is related to macroscopic fiber bundle morphometric change. Although these measures are typically not independent, they can provide insight on different types of WM impairment and have successfully been applied to Alzheimer disease (Mito et al., 2018). In the context of FTD we found that both FD and FC were reduced in similar WM regions which suggest that both fiber atrophy and axonal depletion that are part of the disease.

\section{Strengths and limitations}

This study has several strengths. To our knowledge, this is the first study applying a fixel-based analysis to analyse WM impairments across FTD phenotypes, thus broadening the biological interpretation of WM alterations in the pathophysiology of this disease. There is a growing body of evidence describing WM degeneration in several cortical diseases. However, most studies did not investigate the relationship between specific cognitive domains, whole brain WM properties and structural connectivity. Therefore, our study provided a more complete picture of specific WM tracts involved in core FTD cognitive impairment. Finally, the use an innovative connection-based framework allowing for the quantification of the simultaneous contribution of WM and GM abnormalities on cognitive deficits in FTD, also expanded the knowledge about multimodal contribution to cognition. The main limitations are due to a limited number of subjects and the lack of longitudinal data. Although patients were clinically assessed with the highest standards, the lack of genetic or pathological information precludes any association between the proteins involved in the etiology of FTD, such as tau and TDP-43, and WM fibers. 


\section{Conclusion}

In conclusion, our results support the importance of WM tract disruption to the core cognitive symptoms associated with FTD. While semantic symptoms were mainly dependent on short-range WM fiber disruption, long-range WM fibers damage was the major contributor to executive dysfunction. As large-scale WM tracts, which are particularly vulnerable to vascular disease, were highly associated with executive disfunction, our findings highlight the importance of controlling for risk factors associated with deep white matter disease, such as vascular risk factors, in patients with FTD in order not to potentiate underlying executive dysfunction.

\section{References}

Acosta-Cabronero, J., Patterson, K., Fryer, T. D., Hodges, J. R., Pengas, G., Williams, G. B., \& Nestor, P. J. (2011). Atrophy, hypometabolism and white matter abnormalities in semantic dementia tell a coherent story. Brain. https://doi.org/10.1093/brain/awr119

Agosta, F., Scola, E., Canu, E., Marcone, A., Magnani, G., Sarro, L., ... Filippi, M. (2012). White matter damage in frontotemporal lobar degeneration spectrum. Cerebral Cortex. https://doi.org/10.1093/cercor/bhr288

Agosta, Federica, Galantucci, S., Magnani, G., Marcone, A., Martinelli, D., Antonietta Volontè, M., ... Filippi, M. (2015). MRI signatures of the frontotemporal lobar degeneration continuum. Human Brain Mapping. https://doi.org/10.1002/hbm.22794

Agosta, Federica, Henry, R. G., Migliaccio, R., Neuhaus, J., Miller, B. L., Dronkers, N. F., ... GornoTempini, M. L. (2010). Language networks in semantic dementia. Brain. https://doi.org/10.1093/brain/awp233

Andersson, J. L. R., \& Sotiropoulos, S. N. (2016). An integrated approach to correction for offresonance effects and subject movement in diffusion MR imaging. NeuroImage. https://doi.org/10.1016/j.neuroimage.2015.10.019

Ashburner, J. (2007). A fast diffeomorphic image registration algorithm. NeuroImage. https://doi.org/10.1016/j.neuroimage.2007.07.007

Avants, B. B., Cook, P. A., Ungar, L., Gee, J. C., \& Grossman, M. (2010). Dementia induces correlated reductions in white matter integrity and cortical thickness: A multivariate neuroimaging study with sparse canonical correlation analysis. NeuroImage. 
https://doi.org/10.1016/j.neuroimage.2010.01.041

Bang, J., Spina, S., \& Miller, B. L. (2015). Frontotemporal dementia. The Lancet. https://doi.org/10.1016/S0140-6736(15)00461-4

Bocchetta, M., Gordon, E., Cardoso, M. J., Modat, M., Ourselin, S., Warren, J. D., \& Rohrer, J. D. (2018). Thalamic atrophy in frontotemporal dementia - Not just a C9orf72 problem. NeuroImage: Clinical. https://doi.org/10.1016/j.nicl.2018.02.019

Cairns, N. J., Bigio, E. H., Mackenzie, I. R. A., Neumann, M., Lee, V. M. Y., Hatanpaa, K. J., ... Mann, D. M. A. (2007). Neuropathologic diagnostic and nosologic criteria for frontotemporal lobar degeneration: Consensus of the Consortium for Frontotemporal Lobar Degeneration. Acta Neuropathologica. https://doi.org/10.1007/s00401-007-0237-2

Cocquyt, E. M., Lanckmans, E., van Mierlo, P., Duyck, W., Szmalec, A., Santens, P., \& De Letter, M. (2020). The white matter architecture underlying semantic processing: A systematic review. Neuropsychologia. https://doi.org/10.1016/j.neuropsychologia.2019.107182

Desikan, R. S., Ségonne, F., Fischl, B., Quinn, B. T., Dickerson, B. C., Blacker, D., ... Killiany, R. J. (2006). An automated labeling system for subdividing the human cerebral cortex on MRI scans into gyral based regions of interest. NeuroImage. https://doi.org/10.1016/j.neuroimage.2006.01.021

Dhollander, T., \& Connelly, A. (2016). A novel iterative approach to reap the benefits of multi-tissue $C S D$ from just single-shell $(+b=0)$ diffusion MRI data.

Dhollander, T., Mito, R., Raffelt, D., \& Connelly, A. (2019). Improved white matter response function estimation for 3-tissue constrained spherical deconvolution.

Diehl-Schmid, J., Licata, A., Goldhardt, O., Förstl, H., Yakushew, I., Otto, M., ... Grimmer, T. (2019). FDG-PET underscores the key role of the thalamus in frontotemporal lobar degeneration caused by C9ORF72 mutations. Translational Psychiatry. https://doi.org/10.1038/s41398-019-0381-1

Duffau, H., Gatignol, P., Mandonnet, E., Capelle, L., \& Taillandier, L. (2008). Intraoperative subcortical stimulation mapping of language pathways in a consecutive series of 115 patients with Grade II glioma in the left dominant hemisphere. Journal of Neurosurgery. https://doi.org/10.3171/JNS/2008/109/9/0461

Duffau, H., Gatignol, P., Mandonnet, E., Peruzzi, P., Tzourio-Mazoyer, N., \& Capelle, L. (2005). New insights into the anatomo-functional connectivity of the semantic system: A study using corticosubcortical electrostimulations. Brain. https://doi.org/10.1093/brain/awh423 
Galantucci, S., Tartaglia, M. C., Wilson, S. M., Henry, M. L., Filippi, M., Agosta, F., ... GornoTempini, M. L. (2011). White matter damage in primary progressive aphasias: A diffusion tensor tractography study. Brain. https://doi.org/10.1093/brain/awr099

Greaves, C. V., \& Rohrer, J. D. (2019). An update on genetic frontotemporal dementia. Journal of Neurology. https://doi.org/10.1007/s00415-019-09363-4

Hua, P., Pan, X. P., Hu, R., Mo, X. E., Shang, X. Y., \& Yang, S. R. (2014). Factors related to executive dysfunction after acute infarct. PLoS ONE. https://doi.org/10.1371/journal.pone.0108574

Jefferies, E., Jones, R., Bateman, D., \& Lambon Ralph, M. A. (2004). When does word meaning affect immediate serial recall in semantic dementia? Cognitive, Affective and Behavioral Neuroscience. https://doi.org/10.3758/CABN.4.1.20

Jiskoot, L. C., Bocchetta, M., Nicholas, J. M., Cash, D. M., Thomas, D., Modat, M., ... Rohrer, J. D. (2018). Presymptomatic white matter integrity loss in familial frontotemporal dementia in the GENFI cohort: A cross-sectional diffusion tensor imaging study. Annals of Clinical and Translational Neurology. https://doi.org/10.1002/acn3.601

Kellner, E., Dhital, B., Kiselev, V. G., \& Reisert, M. (2016). Gibbs-ringing artifact removal based on local subvoxel-shifts. Magnetic Resonance in Medicine. https://doi.org/10.1002/mrm.26054

Kinoshita, M., de Champfleur, N. M., Deverdun, J., Moritz-Gasser, S., Herbet, G., \& Duffau, H. (2015). Role of fronto-striatal tract and frontal aslant tract in movement and speech: an axonal mapping study. Brain Structure and Function. https://doi.org/10.1007/s00429-014-0863-0

Knott, R., Patterson, K., \& Hodges, J. R. (2000). The role of speech production in auditory-verbal short-term memory: Evidence from progressive fluent aphasia. Neuropsychologia. https://doi.org/10.1016/S0028-3932(99)00069-X

Ljubenkov, P. A., Staffaroni, A. M., Rojas, J. C., Allen, I. E., Wang, P., Heuer, H., ... Rosen, H. J. (2018). Cerebrospinal fluid biomarkers predict frontotemporal dementia trajectory. Annals of Clinical and Translational Neurology. https://doi.org/10.1002/acn3.643

Mahoney, C. J., Malone, I. B., Ridgway, G. R., Buckley, A. H., Downey, L. E., Golden, H. L., ... Warren, J. D. (2013). White matter tract signatures of the progressive aphasias. Neurobiology of Aging. https://doi.org/10.1016/j.neurobiolaging.2012.12.002

Mahoney, C. J., Ridgway, G. R., Malone, I. B., Downey, L. E., Beck, J., Kinnunen, K. M., ... Warren, J. D. (2014). Profiles of white matter tract pathology in frontotemporal dementia. Human Brain Mapping. https://doi.org/10.1002/hbm.22468 
Mandelli, M. L., Caverzasi, E., Binney, R. J., Henry, M. L., Lobach, I., Block, N., ... Gorno-Tempini, M. L. (2014). Frontal white matter tracts sustaining speech production in primary progressive aphasia. Journal of Neuroscience. https://doi.org/10.1523/JNEUROSCI.3464-13.2014

Martino, J., Brogna, C., Robles, S. G., Vergani, F., \& Duffau, H. (2010). Anatomic dissection of the inferior fronto-occipital fasciculus revisited in the lights of brain stimulation data. Cortex. https://doi.org/10.1016/j.cortex.2009.07.015

Mathotaarachchi, S., Wang, S., Shin, M., Pascoal, T. A., Benedet, A. L., Kang, M. S., ... Rosa-Neto, P. (2016). VoxelStats: A MATLAB Package for Multi-Modal Voxel-Wise Brain Image Analysis. Frontiers in Neuroinformatics. https://doi.org/10.3389/fninf.2016.00020

Matsuo, K., Mizuno, T., Yamada, K., Akazawa, K., Kasai, T., Kondo, M., ... Nakagawa, M. (2008). Cerebral white matter damage in frontotemporal dementia assessed by diffusion tensor tractography. Neuroradiology. https://doi.org/10.1007/s00234-008-0379-5

Meeter, L. H., Kaat, L. D., Rohrer, J. D., \& Van Swieten, J. C. (2017). Imaging and fluid biomarkers in frontotemporal dementia. Nature Reviews Neurology. https://doi.org/10.1038/nrneurol.2017.75

Metzler-Baddeley, C., Mole, J. P., Sims, R., Fasano, F., Evans, J., Jones, D. K., ... Baddeley, R. J. (2019). Fornix white matter glia damage causes hippocampal gray matter damage during agedependent limbic decline. Scientific Reports. https://doi.org/10.1038/s41598-018-37658-5

Mito, R., Raffelt, D., Dhollander, T., Vaughan, D. N., Tournier, J. D., Salvado, O., ... Connelly, A. (2018). Fibre-specific white matter reductions in Alzheimer's disease and mild cognitive impairment. Brain. https://doi.org/10.1093/brain/awx355

Moeller, K., Willmes, K., \& Klein, E. (2015). A review on functional and structural brain connectivity in numerical cognition. Frontiers in Human Neuroscience. https://doi.org/10.3389/fnhum.2015.00227

O’Brien, J. T., Wiseman, R., Burton, E. J., Barber, B., Wesnes, K., Saxby, B., \& Ford, G. A. (2002). Cognitive associations of subcortical white matter lesions in older people. In Annals of the New York Academy of Sciences. https://doi.org/10.1111/j.1749-6632.2002.tb04849.x

Papagno, C. (2011). Naming and the role of the uncinate fasciculus in language function. Current Neurology and Neuroscience Reports. https://doi.org/10.1007/s11910-011-0219-6

Patterson, K., Graham, N., \& Hodges, J. R. (1994). The impact of semantic memory loss on phonological representations. Journal of Cognitive Neuroscience. https://doi.org/10.1162/jocn.1994.6.1.57 
Patterson, K., Nestor, P. J., \& Rogers, T. T. (2007). Where do you know what you know? The representation of semantic knowledge in the human brain. Nature Reviews Neuroscience. https://doi.org/10.1038/nrn2277

Piguet, O., Hornberger, M., Mioshi, E., \& Hodges, J. R. (2011). Behavioural-variant frontotemporal dementia: Diagnosis, clinical staging, and management. The Lancet Neurology. https://doi.org/10.1016/S1474-4422(10)70299-4

Raffelt, D. A., Smith, R. E., Ridgway, G. R., Tournier, J. D., Vaughan, D. N., Rose, S., ... Connelly, A. (2015). Connectivity-based fixel enhancement: Whole-brain statistical analysis of diffusion MRI measures in the presence of crossing fibres. NeuroImage. https://doi.org/10.1016/j.neuroimage.2015.05.039

Raffelt, D. A., Tournier, J. D., Smith, R. E., Vaughan, D. N., Jackson, G., Ridgway, G. R., \& Connelly, A. (2017). Investigating white matter fibre density and morphology using fixel-based analysis. NeuroImage. https://doi.org/10.1016/j.neuroimage.2016.09.029

Raffelt, D., Tournier, J. D., Crozier, S., Connelly, A., \& Salvado, O. (2012). Reorientation of fiber orientation distributions using apodized point spread functions. Magnetic Resonance in Medicine. https://doi.org/10.1002/mrm.23058

Raffelt, D., Tournier, J. D., Fripp, J., Crozier, S., Connelly, A., \& Salvado, O. (2011). Symmetric diffeomorphic registration of fibre orientation distributions. NeuroImage. https://doi.org/10.1016/j.neuroimage.2011.02.014

Raffelt, D., Tournier, J. D., Rose, S., Ridgway, G. R., Henderson, R., Crozier, S., ... Connelly, A. (2012). Apparent Fibre Density: A novel measure for the analysis of diffusion-weighted magnetic resonance images. NeuroImage. https://doi.org/10.1016/j.neuroimage.2011.10.045

Ralph, M. A. L., Jefferies, E., Patterson, K., \& Rogers, T. T. (2016). The neural and computational bases of semantic cognition. Nature Reviews Neuroscience. https://doi.org/10.1038/nrn.2016.150

Ridgway, G. R., Omar, R., Ourselin, S., Hill, D. L. G., Warren, J. D., \& Fox, N. C. (2009). Issues with threshold masking in voxel-based morphometry of atrophied brains. NeuroImage. https://doi.org/10.1016/j.neuroimage.2008.08.045

Rizio, A. A., \& Diaz, M. T. (2016). Language, aging, and cognition: Frontal aslant tract and superior longitudinal fasciculus contribute toward working memory performance in older adults. NeuroReport. https://doi.org/10.1097/WNR.0000000000000597

Rohrer, J. D., Ridgway, G. R., Modat, M., Ourselin, S., Mead, S., Fox, N. C., ... Warren, J. D. (2010). 
Distinct profiles of brain atrophy in frontotemporal lobar degeneration caused by progranulin and tau mutations. NeuroImage. https://doi.org/10.1016/j.neuroimage.2009.12.088

Seelaar, H., Rohrer, J. D., Pijnenburg, Y. A. L., Fox, N. C., \& Van Swieten, J. C. (2011). Clinical, genetic and pathological heterogeneity of frontotemporal dementia: A review. Journal of Neurology, Neurosurgery and Psychiatry. https://doi.org/10.1136/jnnp.2010.212225

Smith, R. E., Tournier, J. D., Calamante, F., \& Connelly, A. (2013). SIFT: Spherical-deconvolution informed filtering of tractograms. NeuroImage. https://doi.org/10.1016/j.neuroimage.2012.11.049

Smith, R. E., Tournier, J. D., Calamante, F., \& Connelly, A. (2015a). SIFT2: Enabling dense quantitative assessment of brain white matter connectivity using streamlines tractography. NeuroImage. https://doi.org/10.1016/j.neuroimage.2015.06.092

Smith, R. E., Tournier, J. D., Calamante, F., \& Connelly, A. (2015b). The effects of SIFT on the reproducibility and biological accuracy of the structural connectome. NeuroImage. https://doi.org/10.1016/j.neuroimage.2014.10.004

Staffaroni, A. M., Ljubenkov, P. A., Kornak, J., Cobigo, Y., Datta, S., Marx, G., ... Rosen, H. J. (2019). Longitudinal multimodal imaging and clinical endpoints for frontotemporal dementia clinical trials. Brain. https://doi.org/10.1093/brain/awy319

Tournier, J.-D., \& , F. Calamante, and a. C. (2010). Improved probabilistic streamlines tractography by 2 nd order integration over fibre orientation distributions. Ismrm.

Tournier, J. D., Calamante, F., \& Connelly, A. (2007). Robust determination of the fibre orientation distribution in diffusion MRI: Non-negativity constrained super-resolved spherical deconvolution. NeuroImage. https://doi.org/10.1016/j.neuroimage.2007.02.016

Tournier, J. D., Calamante, F., Gadian, D. G., \& Connelly, A. (2004). Direct estimation of the fiber orientation density function from diffusion-weighted MRI data using spherical deconvolution. NeuroImage. https://doi.org/10.1016/j.neuroimage.2004.07.037

Tournier, J. D., Smith, R., Raffelt, D., Tabbara, R., Dhollander, T., Pietsch, M., .. Connelly, A. (2019). MRtrix3: A fast, flexible and open software framework for medical image processing and visualisation. NeuroImage. https://doi.org/10.1016/j.neuroimage.2019.116137

Tournier, J. D., Yeh, C. H., Calamante, F., Cho, K. H., Connelly, A., \& Lin, C. P. (2008). Resolving crossing fibres using constrained spherical deconvolution: Validation using diffusion-weighted imaging phantom data. NeuroImage. https://doi.org/10.1016/j.neuroimage.2008.05.002

Turken, A. U., Whitfield-Gabrieli, S., Bammer, R., Baldo, J. V., Dronkers, N. F., \& Gabrieli, J. D. E. 
(2008). Cognitive processing speed and the structure of white matter pathways: Convergent evidence from normal variation and lesion studies. NeuroImage. https://doi.org/10.1016/j.neuroimage.2008.03.057

Tustison, N. J., Avants, B. B., Cook, P. A., Zheng, Y., Egan, A., Yushkevich, P. A., \& Gee, J. C. (2010). N4ITK: Improved N3 bias correction. IEEE Transactions on Medical Imaging. https://doi.org/10.1109/TMI.2010.2046908

Veraart, J., Novikov, D. S., Christiaens, D., Ades-aron, B., Sijbers, J., \& Fieremans, E. (2016). Denoising of diffusion MRI using random matrix theory. NeuroImage. https://doi.org/10.1016/j.neuroimage.2016.08.016

Villain, N., Desgranges, B., Viader, F., de la Sayette, V., Mezenge, F., Landeau, B., ... Chetelat, G. (2008). Relationships between Hippocampal Atrophy, White Matter Disruption, and Gray Matter Hypometabolism in Alzheimer's Disease. Journal of Neuroscience. https://doi.org/10.1523/jneurosci.1392-08.2008

Villain, Nicolas, Fouquet, M., Baron, J. C., Mézenge, F., Landeau, B., De La Sayette, V., ... Chételat, G. (2010). Sequential relationships between grey matter and white matter atrophy and brain metabolic abnormalities in early Alzheimer's disease. Brain. https://doi.org/10.1093/brain/awq203 Vinokur, L., Zalesky, A., Raffelt, D., Smith, R. ., \& Connelly, A. (2015). A Novel Threshold-Free Network-Based Statistics Method: Demonstration using Simulated Pathology. In Organization for Human Brain Mapping (p. 4144). Hawaii.

Whiteside, D. M., Kealey, T., Semla, M., Luu, H., Rice, L., Basso, M. R., \& Roper, B. (2016). Verbal Fluency: Language or Executive Function Measure? Applied Neuropsychology:Adult. https://doi.org/10.1080/23279095.2015.1004574

Whitwell, J. L., Avula, R., Senjem, M. L., Kantarci, K., Weigand, S. D., Samikoglu, A., ... Jack, C. R. (2010). Gray and white matter water diffusion in the syndromic variants of frontotemporal dementia. Neurology. https://doi.org/10.1212/WNL.0b013e3181d9edde

Xia, M., Wang, J., \& He, Y. (2013). BrainNet Viewer: A Network Visualization Tool for Human Brain Connectomics. PLoS ONE. https://doi.org/10.1371/journal.pone.0068910

Young, V. G., Halliday, G. M., \& Kril, J. J. (2008). Neuropathologic correlates of white matter hyperintensities. Neurology. https://doi.org/10.1212/01.wnl.0000319691.50117.54

Yu, J., \& Lee, T. M. C. (2019). The longitudinal decline of white matter microstructural integrity in behavioral variant frontotemporal dementia and its association with executive function. 
bioRxiv preprint doi: https://doi.org/10.1101/2020.05.01.064576; this version posted May 2, 2020. The copyright holder for this preprint (which

was not certified by peer review) is the author/funder, who has granted bioRxiv a license to display the preprint in perpetuity. It is made available under aCC-BY-NC-ND 4.0 International license.

Neurobiology of Aging. https://doi.org/10.1016/j.neurobiolaging.2018.12.005

Zhang, Y., Schuff, N., Du, A. T., Rosen, H. J., Kramer, J. H., Gorno-Tempini, M. L., ... Weiner, M. W. (2009). White matter damage in frontotemporal dementia and Alzheimers disease measured by diffusion MRI. Brain. https://doi.org/10.1093/brain/awp071 